NIST Advanced Manufacturing Series 100-41

\title{
Best Practices for the Integration of Collaborative Robots into Workcells Within Small and Medium-Sized Manufacturing Operations
}

\author{
John Horst \\ Jeremy Marvel \\ Elena Messina
}

This publication is available free of charge from:

https://doi.org/10.6028/NIST.AMS.100-41 


\title{
Best Practices for the Integration of Collaborative Robots into Workcells Within Small and Medium-Sized Manufacturing Operations
}

\author{
John Horst \\ Jeremy Marvel \\ Elena Messina \\ Intelligent Systems Division \\ Engineering Laboratory \\ National Institute of Standards and Technology \\ Gaithersburg, Maryland 20899
}

This publication is available free of charge from:

https://doi.org/10.6028/NIST.AMS.100-41

May 2021

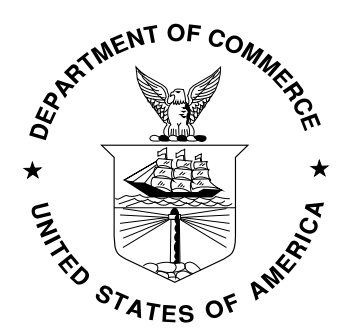

U.S. Department of Commerce

Gina M. Raimondo, Secretary

National Institute of Standards and Technology James K. Olthoff, Performing the Non-Exclusive Functions and Duties of the Under Secretary of Commerce for Standards and Technology \& Director, National Institute of Standards and Technology 
Certain commercial entities, equipment, or materials may be identified in this document in order to describe an experimental procedure or concept adequately. Such identification is not intended to imply recommendation or endorsement by the National Institute of Standards and Technology, nor is it intended to imply that the entities, materials, or equipment are necessarily the best available for the purpose.

National Institute of Standards and Technology Advanced Manufacturing Series 100-41

Natl. Inst. Stand. Technol. Adv. Man. Ser. 100-41, 21 pages (May 2021)

This publication is available free of charge from: https://doi.org/10.6028/NIST.AMS.100-41 


\begin{abstract}
This document provides guidance for small- and medium-sized manufacturers to identify the key factors and performance requirements of their workcells that are best served by collaborative robot (or "cobot") integration. This guidance is provided based on current industry practices, as identified through a broad survey of manufacturers currently leveraging cobot technologies in their production lines. This document starts with basic and quick cobot integration methods and moves to more time-consuming but more accurate cobot integration methods.
\end{abstract}

\title{
Key words
}

Collaborative robot; cobot; cobot integration; complexity-impact method; key-elements weighting method; manufacturing; Manufacturing Extension Partnership; manufacturing workcells; past experience-based method; small to medium-sized manufacturers (SMMs).

\section{Table of Contents}

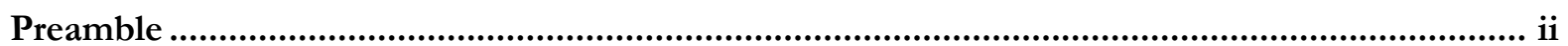

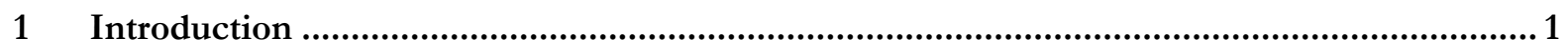

2 What is the workcell within a plant that is most suitable for cobot integration?......................2

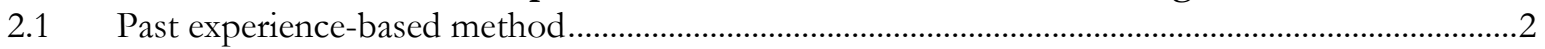

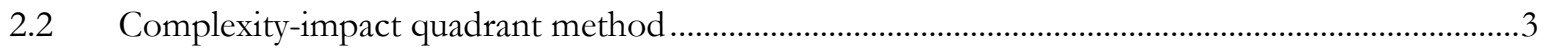

$2.3 \quad$ Key-elements weighting method ....................................................................................................

3 What is the best cobot system for the chosen workcell? .........................................................6

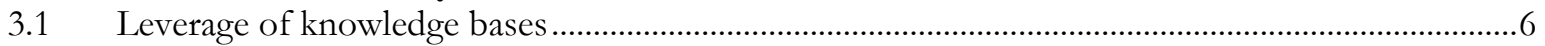

3.2 Task-relevant specifications-based decision making .....................................................................6

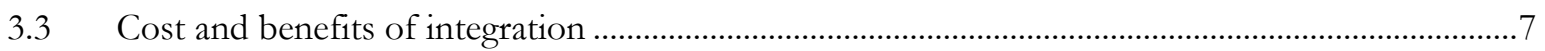

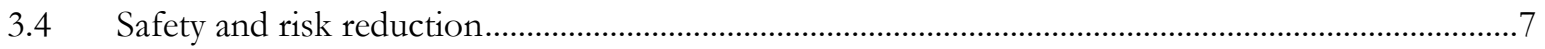

4 What is the best tactic for cobot system integration? ..............................................................8

4.1 Leveraging vendor support and maintenance ................................................................................

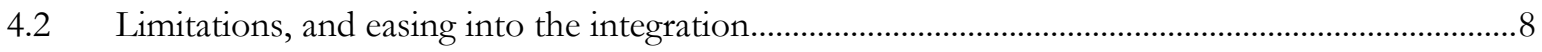

4.3 Capabilities and benefits models ..........................................................................................

5 What are the latest requirements, benefits, barriers, knowledge, best-practices, challenges, and dangers for choosing and integrating collaborative robotic systems into different types of SMM workcells?.

\section{Appendices}

Appendix A: Key-elements weighting approach

Appendix B: An example case study for the Key-elements weighting approach

List of Figures

References 


\section{Preamble}

In the United States, a significant portion of manufacturers are considered "small." Per the U.S. Bureau of Labor Statistics, in 2017, 94\% of all manufacturing firms had fewer than 500 employees ${ }^{1}$; and $75 \%$ of these small manufacturers had fewer than 20 employees. More broadly speaking, the U.S. Small Business Administration estimates that, in 2020, all small businesses (including both manufacturers and nonmanufacturers $)^{2}$ represented $99.9 \%$ (31.7 million) of all businesses in the U.S., accounting for $47.1 \%$ of the U.S. workforce (60.6 million people). Small businesses also comprise over $97 \%$ of all exporters in the U.S., generating $32 \%$ of its total $\$ 1.5$ trillion in exports.

Clearly, the economic engine that is U. S. manufacturing is powered by small enterprises. And yet, small- and medium-sized manufacturing is being threatened by market forces, struggling to compete against foreign industries and domestic economic factors. To remain competitive (and in some cases, simply survive), many small manufacturers are turning to automation. These small organizations typically have limited resources or capacity for adopting new technologies and automation. They face challenges in incorporating collaborative robots into their operations due to lack of expertise, of sufficient knowledge of the capabilities and limitations of robots, and of unbiased guidance for making investment and technology decisions.

The National Institute of Standards and Technology (NIST) Measurement Science for Manufacturing Robotics (MSMR) program ${ }^{3}$ is developing measurement science, standards, and test methods that advance manufacturing robotic system performance, collaboration, agility, autonomy, safety, and ease of implementation to enhance U.S. innovation and industrial competitiveness. The program is particularly focused on enabling effective utilization of robotic technologies by small and medium manufacturers (SMMs). This includes developing understanding of unique requirements and challenges that SMMs have in implementing robotic systems.

In addition to the NIST laboratories, the NIST Hollings Manufacturing Extension Partnership (MEP) is tasked with the mission of enhancing the productivity and technological performance of U.S. manufacturing. NIST MEP is a public-private partnership and provides federal funding for a national network of 51 MEP centers located in all 50 states and Puerto Rico, with over 1,400 trusted advisors and experts at approximately 375 MEP service locations, providing any U.S. manufacturer with access to resources they need to succeed. The MSMR Program partners with NIST MEP headquarters, centers, and network members to gain knowledge about SMM needs and to share MSMR-developed resources aimed at reducing the barriers to implementing robotics.

The MSMR program is studying available information about successful adoption of robotics by SMMs. This investigation includes staying up to date with information presented by robot manufacturers, systems integrators, and users. First-hand experiences of SMMs, disseminated through the MEP network, are particularly valuable. These experiences form the basis for this report.

\footnotetext{
${ }^{1}$ In the United States, a generally accepted upper limit of 500 employees is required to be considered a "small" business. Note that this is the number of employees inside the United States and its territories and does not consider the global employee count for the company. In some instances, if the company has smaller subsidiaries or independently operated branches, each of these branches may be assessed independently.

${ }^{2}$ In 2020, small business manufacturers were the sixth largest employer in the U.S., with health care and social assistance being the largest category for employment.

${ }^{3}$ https://www.nist.gov/programs-projects/measurement-science-manufacturing-robotics
} 


\section{Introduction}

This document offers best practices for successfully integrating collaborative robots ("cobots") 4 into the production processes of small- and medium-sized manufacturers (SMMs) based on industry recommendations, feedback from successful integrations, and descriptions of the current state of practice. Knowledge was gathered from robotics experts from a few representative MEP centers within the NIST MEP network in the USA 5 , with each of these MEP centers having their own unique manufacturing operations and focuses. Collectively, the NIST MEP is a public-private partnership, and is intended to service collaborations at the federal, state, and local levels, enhancing productivity, technology performance, and economic competitiveness of U.S. manufacturers. The 51 total MEP centers (one for each of the 50 states, and Puerto Rico) are largely operated independently, but are part of a common network facilitated by NIST MEP headquarters in Gaithersburg, MD.

For this assessment, the authors consulted with a number of MEP workers from five MEP centers to gain industry insights. Interactions with SMMs and MEP workers consisted of communications via in-person meetings, email exchanges, and telephone conversations. These interactions were open-format discussions, largely driven by the following targeted questions for the MEP experts:

- What is the workcell within the plant that is most suitable to cobot integration?

- What is the best cobot system for the chosen workcell?

- What is the best tactic for cobot system integration?

- What are the latest requirements, benefits, barriers, knowledge, best-practices, challenges, and dangers for choosing and integrating collaborative robotic systems into different types of SMM workcells?

The responses to these questions were collected from MEP experts and then collated by workers from the NIST Engineering Laboratory (EL). In the following sections, we present and summarize this feedback from the NIST MEP robotics experts, as they responded to the four aforementioned questions listed below.

\footnotetext{
${ }^{4}$ Colloquially, a "collaborative robot" is an robot that has specific safety functions that are intended to enable the robot to operate safely and effectively near human workers, thus avoiding the expense, constraint, and inconvenience of robot workcells required to be in a protective cage for safety reasons. Ultimately, the term is a misnomer, as there are no collaborative robots, only collaborative robot applications. On the downside, cobots are generally smaller, typically carry payloads of lesser weight than a traditional robot. A safety risk assessment is required for the total robot system per application. For more details of these safety functions and the means by which collaborative robot safety can be effectively applied, consult with robot/cobot safety standards such as RIA TR15.606-2016 Collaborative Robots and RIA TR15.806-2018 Guide to Testing Pressure and Force in Collaborative Robot Applications.

${ }^{5}$ Surveys from TechSolve, Inc. (Cincinnati, Ohio, USA), Cleveland Manufacturing Advocacy and Growth Network (MAGNET) (Cleveland, Ohio, USA), Pittsburgh Catalyst Connections (Pittsburgh, Pennsylvania, USA), South Dakota Manufacturing \& Technology Solutions (Sioux Falls, South Dakota, USA), and Dayton FASTLANE (Dayton, Ohio, USA)
} 


\section{What is the workcell within a plant that is most suitable for cobot integration?}

When considering candidate workcells for automation leveraging cobots, the MEP robotics experts responded by listing criteria for workcell analysis and cost-benefit analysis. In general, SMMs want to know which workcells will deliver a quick, substantial, and enduring return on investment (ROI) when integrating a cobot system into those workcells. For most manufacturers, ROI is assessed as the mean time to recovering the initial cost of system integration through gained profits $^{6}$. Leading integrator companies are known to produce a full ROI in about one year for several workcell types. Integrators are typically highly capable and trusted. An integrator can perform technology demonstrations to help identify possible solutions before investing in a system design. This process will save manufacturers time and money ${ }^{7}$. Recently, 33\% of manufacturers surveyed indicated "searching for [robotics] suppliers and integrators" as an area of interest for them ${ }^{8}$, implying that a significant segment of SMMs are considering engaging someone in the robotics supply chain to address specific production needs. For those SMMs that do not have in-house robotics expertise, many will choose to engage integrators to develop targeted solutions for manufacturing challenges.

There are, of course, a myriad of reasons to employ an integrator. The most common of these reasons is that integrators have the tools and expertise necessary to design, construct, deploy, and support robotic workcells. For SMMs that do not have such capabilities, hiring an integrator can be the most expeditious and efficient route to introducing robots into an existing or new workflow. Sometimes, it is simply easier to have an integrator take care of the robotics elements rather than establishing in-house expertise, particularly if there are not enough personnel available to take on the role. And, by accepting the contract, the integrator often assumes the responsibility and liability if specific production goals, e.g., overall equipment efficiency, production throughput, or reductions in waste or downtime, are not achieved.

However, there are just as many reasons that SMMs may choose not to employ a robot system integrator. For example, engaging an integrator can be expensive and too costly for the SMM. Furthermore, experienced integrators may not be available in the local region of the SMM.

But if an SMM does choose to integrate a cobot into their operation by themselves, it is obviously best to choose a workcell having a high probability of substantial success.

Several different methods for assessing a workcell's suitability for cobot integration are presented below, starting with methods that are time-consuming but thorough, to methods that are timesaving but less thorough. It is expected that these several methods would be performed by a small team of manufacturing experts, including SMM managers, SMM workers, system integrators, and MEP experts.

\subsection{Past experience-based method}

A recent industry survey ${ }^{9}$ of manufacturers estimates the degree to which certain manufacturing robotintegrated workcells have benefitted ${ }^{10}$ those manufacturers, as illustrated in Figure 1. SMMs can use this information to guide their choice of a promising workcell.

\footnotetext{
${ }^{6}$ An alternative metric for evaluating return on investment is to assess the projected net income versus the initial investment in resources over the life of the equipment. Larger ROIs are considered favorable indicators of long-term value of investing in equipment or services, but do not always consider the factors of maintenance costs, impacts on availability of equipment or other resources, or the generation of waste.

${ }^{7}$ Manufacturers often consider time and money as one and the same

${ }^{8} 2018$ survey from TechSolve, Inc.

9 TechSolve, Inc.

${ }^{10}$ Cobot integration improved their cumulative benefits (speed, ROI, accuracy, waste reduction) by the amount of percentage points indicated
} 


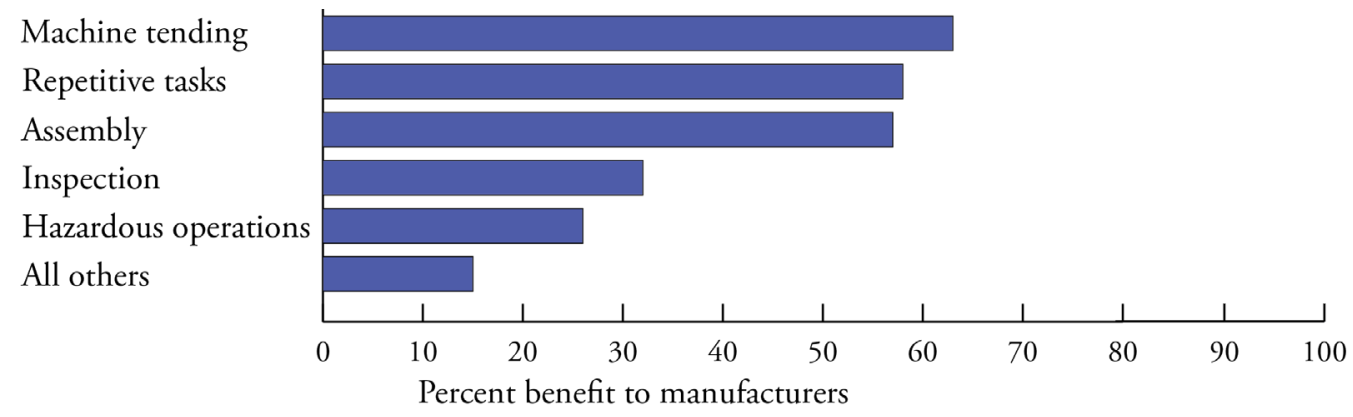

Figure 1: Robot-integrated workcell types with percent benefit to the manufacturers

In another survey ${ }^{11}$, manufacturers said that robots have worked successfully with the following workcell types: part loading and unloading, cleaning of parts, pick-place-orient-pack parts, inspection, packaging, welding, assembly, and any repetitive operation. It is also argued that workcell tasks that are boring and/or dangerous should be given high consideration for robot automation. SMMs could make use of these proven results to make a simple decision on what workcell to select for their first or next cobot system implementation. More importantly, SMMs can use these assessments and apply the complexity-impact approach (described in Section 2.2) or the performance element weighting approach (described in Section 2.3), starting with the highest valued workcells.

\subsection{Complexity-impact quadrant method}

The complexity-impact quadrant method ${ }^{12}$ is a decision-making tool for choosing a workcell well suited for cobot integration. The method seeks to balance "simplicity" for the company (e.g., low setup time and cost performance) with "high impact" for the company (e.g., improved performance and higher ROI) for each workcell. This method is illustrated in Figure 2.

One can see (in Figure 2) thatpriority is given to simplicity over impact, as indicated by the hierarchy of workcell choices (1 1 st $2^{\text {nd }}$, and $3^{\text {rd }}$. Cobot unsuitability is reserved for workcells with some combined level of "low impact" and "high complexity." This method may be leveraged for deciding if and when to design and install new workcells, or if and when to upgrade or repurpose existing workcells to expand current manufacturing capabilities.

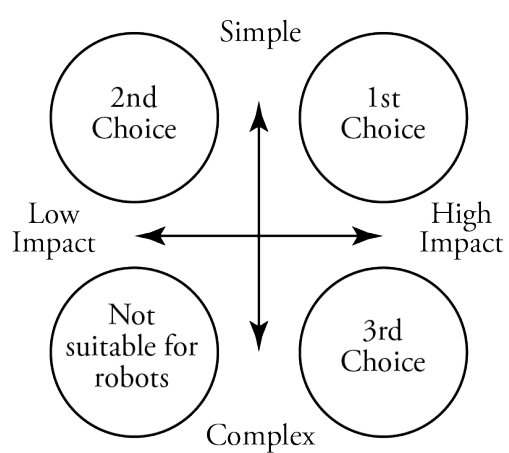

Figure 2: Complexity-impact quadrant

The characterization of simple versus complex and high impact versus low impact may be prone to subjectively qualitative assessments and may therefore vary based on the stakeholders' requirements and corporate priorities. However, more objectively quantitative metrics may also be leveraged based on select key performance indicators:

- Quantitative application criteria for complexity includes metrics such as task complexity (e.g., the number of processing steps required to complete the task), part manipulation difficulty, process inconsistency, part variation, required precision, and integration complexity.

\footnotetext{
11 TechSolve, Inc.

12 Robotiq (robotiq.com)
} 
- Quantitative application criteria for impact includes metrics such as finished part value, stock value, wellbeing and safety (e.g., ergonomics and the mitigation of risk), labor savings, work-in-process, number of parts to be manufactured (e.g., leveraging automation for large runs of identical parts versus smaller, one-off batches), cycle time, quality, production cost, waste reduction, and return on investment.

SMMs can assess simplicity and impact levels using each of the application criteria suggested above and select workcells with the highest benefit. In some cases, a workcell with very high impact will and should be selected, even if the workcell operation is very complex, and visa-versa, a workcell with high simplicity, might be selected, even if the workcell had a demonstrably low impact.

Another equally important perspective is how workforce utilization relates to the complexity-impact quadrant method. Automating processes that are dull, dirty, and/or dangerous are commonly considered as good candidates for automation ${ }^{13}$, however, such processes can be "simple," "high impact," both, or neither. Certain high impact and simple workcells that are challenging for automation may be easy for humans. In that case, a manufacturer may choose to employ a human worker rather than a cobot in those workcells.

Therefore, integrating human workers may produce simplicity and high impact, based on the type of workcell. Furthermore, workers applying their skills to more value-added workcells, and automation applied to less value added workcells, may also produce simplicity and high impact.

\subsection{Key-elements weighting method}

The key-elements weighting method estimates the importance of a much broader set of key elements than the methods described in Sections 2.1 and 2.2. When a SMM is considering how to integrate a cobot-based system into one or more candidate workcells, this approach would be the most thorough compared to the previous methods but will likely take more time and effort for an expert, or a team of experts, to complete. This method should be selected if management is most concerned about selecting the best workcell to support the manufacturing application, (e.g., if success is critical and this is the first attempt at cobot automation).

The key elements are organized into the following categories: performance elements, usage elements, workforce elements, financial elements, and miscellaneous elements. The key elements are listed and described in Appendix A. An example of a couple of performance elements are "reach of the cobot adequate to the task" and "cobot arm speed adequacy to the task." If time is of the essence, elements in Appendix A that are considered of lesser importance can be trimmed from the assessment.

Certain elements can lead to a stalemate, for instance, if a certain level of performance is required, but cannot be performed by any known cobot, then a cobot is not at all suitable for integration into that workcell. For example, if the performance element is the maximum cost of the cobot, and that cost is lower than all existing cobot models of the required size and performance, then no cobot is suitable for integration into that workcell.

To make a good assessment for each workcell, the manufacturing experts need a reasonably accurate estimate of weights for each key element in each candidate workcell, based on that element's relative importance, possibly allowing for both negative and positive weightings. Then all weights are summed up to get a final score for each candidate workcell. For example, for certain tasks in a workcell, a human worker may provide better performance and cost-effectiveness than a cobot system. The elements for weighting ${ }^{14}$, grouped into four categories, are performance, usage, financial, and miscellaneous. Manufacturers/integrators might ignore

\footnotetext{
13 https://www.robotics.org/blog-article.cfm/How-Robots-Are-Taking-on-the-Dirty-Dangerous-and-Dull-Jobs/209

${ }^{14}$ The number of weight values need be no more than 5 , for example, $\{-2,-1,0,1,2\}$ or $\{-1,0,1,2\}$. Fewer weights will save time in the assessment, and since additional weight values might be meaningless, given the uncertainty of estimates.
} 
several of these elements and add other elements, as they see fit. To clarify the approach, we construct a hypothetical example exercise in Appendix B for calculating final scores for a given workcell. 


\section{What is the best cobot system for the chosen workcell?}

When inquired about the means by which cobot systems are chosen, the survey respondents responded with several performance criteria with regards to the robots that were most critical for their particular applications. In some instances, the answer to the question was more pragmatic, and focused more on whether a cobot should be used, leveraging the risks against the rewards of introducing automation into legacy workcells or processes. While many cited safety as a driving factor for deploying cobot technologies, others also indicated that performance and functionality requirements must still be met.

The range of survey responses largely fall into four categories:

1. Leveraging existing knowledge bases to assist with the selection of cobots,

2. Weighing the robots' specifications against the task requirements,

3. Factoring costs and benefits of integrating cobots into workcells, and

4. Safety and risk reduction.

\subsection{Leverage of knowledge bases}

When considering making changes to processes or introducing transformative new capabilities into the facility, knowing what you know and what you do not may be a distinguishing element between financial success and ruin. Sometimes, conferring with external experts may be the best decision, especially when one is not fully aware of the capabilities and limitations of robotic solutions.

Specific responses to the survey include:

- From a state MEP or an integrator standpoint, many SMMs do not perform ROIs, in part because most of them do not perform detailed accounting at all, which would argue for the value of implementing even the most basic cobot integration suitability methods, as described in Section 1.

- Consider employing a system integrator, distributor, or reseller for choosing the most suitable cobot system and software. They are commonly experienced, capable, and trustworthy, and will perform demonstrations, all of which might save manufacturers money and/or time.

\subsection{Task-relevant specifications-based decision making}

Selecting a robot that is not capable of actually performing the task to be automated can be disastrous. Though some robots may look similar, they can have vastly divergent capabilities. Factors such as work volume, lift capacity, repeatability, and event package options (e.g., software packages for assembly, painting, and surface finishing) can vary significantly between manufacturers and even within models of a product line.

Specific responses to the survey include:

- Discover and review the speed and accuracy of all cobot systems and models relevant to your manufacturing use cases.

- Determine cobot system repeatability with respect to the task.

- Quantify all payload, speed, and accuracy limits in cobot systems under review, including maximum loads at all positions and orientations required by the chosen task, to ensure that all candidate cobot systems meet those requirements. 


\subsection{Cost and benefits of integration}

As anyone who has ever had to manage change can attest, almost all decisions have a cost associated with them. Being aware of the capabilities and limitations of robotics and automation can be the key to success, avoid unnecessary costs, and mitigate disruption and downtime of vital production lines due to poor estimates of integration and commissioning. Similarly, mindfully investing in cobots and automation also includes investing in infrastructure, personnel, and future applications.

Specific responses to the survey include:

- Do not go blindly into an automation process and perform the cobot automation in the same way the human operator did it.

- Calculate the human involvement level that satisfies efficiency and quality requirements.

- Estimate operator costs from the calculated level of human involvement.

- The initial cost of integration and disruption of production can be minimized if no safety cell is required, as is typical when a traditional robot is employed instead of a cobot.

- The nature and adequacy of the support for any cobot system product is the main differentiator for implementation and ROI success, so make sure that appropriate product support is implemented.

- Consider human/cobot interaction if it can be shown to be a faster-better-cheaper option.

\subsection{Safety and risk reduction}

One of the principal defining task characteristics that factors into the decision to leverage cobots is the safety of the system. Cobots are selected not because of their stellar physical prowess, but because they are designed specifically to work safely around people. Because "collaborative robots" do not actually exist—but rather only robots used in collaborative applications - it is vital that the chosen cobots have the safety functions necessary to reduce residual risks. Some applications may require robots with power and force limiting, while other applications would be better candidates for speed and separation monitoring safety functions. As such, the selection of the robot becomes an extension of the risk assessment.

Specific responses to the survey include:

- Quantify the safety of all cobot systems under review for the chosen task. Just because you are using a cobot does not mean that the workcell is completely safe for humans in the workcell

- Even though cobots typically share a workspace with employees, determine the safety requirements with the cobot and the human in the work cell with a risk assessment (which may call for expensive fencing/light curtains).

- Quantify all safety precautions required by the chosen task, since it can result in low operating speeds or multiple stops when a human is detected in the workcell. 


\section{What is the best tactic for cobot system integration?}

When asked about tactics for cobot integration, respondents were largely consistent in their assessments of the importance of cost and returns on investment for selecting and integrating cobots for their application needs. These may be either direct or indirect costs, and even subtle impacts on long-term ROI. The following principal sources of cost were identified by the respondents:

- Cost and availability of support and maintenance for installed robots by the integrators and/or vendors,

- Leveraging the ease of integration and support versus the complexity of the task (including potential hidden costs, necessary infrastructure/technology upgrades), and

- Realizing the capabilities and limitations of the robots may necessitate compromise to realize integration benefits.

\subsection{Leveraging vendor support and maintenance}

Recognizing that robots do not support themselves, the costs and challenges associated with integrating cobots into a workcell may necessitate taking steps to ensure external support is available if something happens to the robot investment. For example, one of the costs associated with contracting an integrator is that the integrator often shoulders the responsibility of maintenance and ready access to customer support. Understand what is and is not included in this agreement is therefore important.

Specific responses to the survey include:

- Consider employing a systems integrator, distributor, or reseller for cobot systems integration.

- Cobot system vendor or MEP support and service is essential, particularly in poorer or more sparsely populated states, since the economic climate is not amenable for integrator companies to thrive in.

- Quantify the level and cost of support from resellers or integrators. The latter is the most common and resellers generally have a small staff of integrators.

- Consider that a cobot system integrator may not supply full services within the cost point of an SMM. If so, MEP-supported models might work better, as described in Section 1.

\subsection{Limitations, and easing into the integration}

Just as when considering the capabilities and limitations when selecting cobots, an assessment of the task to be automated is frequently necessary to maintain realistic expectation of the impacts of integrating cobot workcells. This may include reviewing assumptions regarding frequency of operator inputs, and a thorough evaluation of the collaborative application to account for all updates, upgrades, or substitutions necessary to support cobots in the workcell.

Specific responses to the survey include:

- Consider implementing cobot operations in workcells that do not require decision making.

- Identify additional requisite elements relating to the cobot such as, mounting, fixturing, employing end effectors, additional cobot arms, vision systems, assembly, and training (in system setup, operation, and maintenance).

- Chart all information exchange from system to system, e.g. cobot to machine tool, machine tool to cobot, cobot to conveyor, etc. 


\subsection{Capabilities and benefits models}

And, finally, a number of respondents identified strategic deployment of cobots to get the biggest ROI with the least amount of effort. This may include designing and configuring the robot workcell to operate as close to peak efficiency given the robot's capabilities, selecting tasks for automation that are may uniquely require cobot solutions, and even tuning behaviors and capabilities until a desired performance is achieved.

Specific responses to the survey include:

- Describe the workcell process flow by identifying each step in the process, including inputs and outputs per procedure; then specify where the cobot would be needed in process, which can be used as a starting point for a basic understanding of what to program.

- Consider that cobots are slower and generally less accurate than regular robots.

- Define material flow and tolerance of all parts.

- Record time and visuals of the current state without a cobot. After cobot integration, record time and visuals of the automated state for optimization, waste reduction, and to calculate ROI. 


\section{What are the latest requirements, benefits, barriers, knowledge, best-practices, challenges, and dangers for choosing and integrating collaborative robotic systems into different types of SMM workcells?}

When asked about specific challenges and opportunities with integrating cobot workcells, survey respondents shared similar hopes and doubts. Many expressed concerns regarding the state and reliability of cobot technologies, while others cited impacts on personnel and equipment as factors that need addressing. There exists an underlying expectation that cobots will perform as collaborative tools for skilled labor, and not as replacements for the workforce. Because people are the lifeblood of SMMs, cobots are brought in as collaborative tools, and therefore must be consistent in performance, and usable by the workforce.

The survey respondents' feedback is summarized below:

- Benefits:

○ Reduces workplace hazards

- Improves process/task consistency

- Increases flexibility

- Extends workforce skills, capability, and flexibility

- Barriers:

- Cobot system may not be adaptable to a wide enough variety of workcells

- Transition time to accommodate two or more batch types.

$\circ$ Vision system reprogramming is time consuming

- Multiple incompatible programming languages and connectors are costly

- Unsafe conditions, e.g., integrating a cobot into a highly combustible environment

$\circ$ In a union shop it may be harder to implement cobots if workers are replaced

- Finding the right technical person to keep the cobot system running successfully

- Risks:

- Hardware and software changes to programs and systems

- Many of these technologies (cobots, vision, special gripper systems, and other technologies) are being developed by start-ups and smaller companies. This leaves a potential for some of these vendors to go out of business, merge, or be taken over by a larger organization. Concern would be for longterm support related to service, buying similar products, etc. 


\section{Acknowledgements}

Much of the content in this document was developed from the input and experience from the following organizations and individuals: TechSolve (Mark Huffman, Susan Moehring, Radu Pavel), Catalyst Connections (Matt Minner), South Dakota Manufacturing \& Technology Solutions (Keith Deibert, Wes Kelly), and the Cleveland MAGNET (Mike Pintz, Joe Work, Mike O’Donnell, Nick McClellen). The authors express sincere gratitude to these organizations and individuals. 


\section{Appendix A: Key-elements weighting approach}

A community of experts in the manufacturing plant is recommended to assign weights appropriate for each element in each candidate workcell. For each candidate workcell, the experts assign weights to all subelements (e.g., element A3 $=-1$, or element B7 = 2) for a candidate workcell, then do the same for all the rest of the candidate work cells. We recommend keeping the number of weights small, e.g. $\{-1,0,1,2\}$ or even $\{-$ $1,0,1\}$, since there are so many elements. Also, just eliminate elements that are not relevant. Otherwise, score everything, both good and bad, since the goal is to choose the best workcell for your operation. What follows now are the key elements for performance, usage, workforce, financial, and miscellaneous elements.

A: Key elements for performance, e.g., padding on cobot arms and eye safety might still be required with a cobot, along with safety measures such as fencing/light curtains, which can also result in low operating speeds or multiple stops, if humans are detected in certain parts of the workcell.

1: Safety for and desirability from humans to work at the workcell ${ }^{15}$

2: Number of operations per unit time

3: Available shop floor footprint for the cobot system

4: Reach of the cobot adequate to the task or tasks

5: Accuracy of cobot arm motion throughout the entire reach of the cobot

6: Repeatability of cobot arm motion throughout the entire reach of the cobot, and it's relevance to the $\operatorname{task}^{16}$

7: Maximum payloads allowed per cobot compared to human accuracy/precision (including humans with exoskeletons) per the required payloads

8: Cobot arm speed adequacy to the task

9: Cobot system longevity

10: Chosen end effector (gripper) grasping and moving effectiveness for given part types or material

11: Cobot works for many types of use cases (e.g., material handling, machine tending)

12: Minimum and maximum part feature size resolution of cobot gripper and tools satisfies the requirements of the workcell tasks

13: Ability to adapt to widely and frequently varying production requirements, i.e., system agility

B: Key elements for usage

1: System setup, cobot integration, reconfiguration, programming, reprogramming, programming complexity, hardware changeovers, relocation, testing, maintenance, handling multi-product changeovers and mixes, and use (programming or reprogramming is typically given high importance)

2: Even though cobot technology is relatively simple to integrate and program, there is the cost of learning and integrating one or more computing languages, computer programs, and hardware interfaces, e.g., programable logic controllers (PLC), high-definition multimedia interface (HDMI), universal serial bus (USB), and software languages

3: Human decision-making requirements in the workcell

4: If vision sensors are required on or near the cobot: requirements and cost of vision system setup and reprogramming

5: Duration of downtime prior to successful workcell operation

6: The proposed cobot system automates more workcells than one

7: Robust with respect to damage, e.g., are the parts under test expensive and fragile?

C: Key elements for workforce

${ }^{15}$ This would include the undesirableness of having a human in the workcell (e.g., workcell activities that are dull, dirty, or dangerous), or a lack of interest on the part of humans to work in the workcell, e.g., because the work is tedious, has ergonomic issues, etc.

${ }^{16} 0.1 \mathrm{~mm}$ or 0.004 inch repeatability is typical 
1: Technical suitability of manual operators for maximum desired production volumes for the candidate workcell

2: Danger to workers of the candidate workcell operation

3: Boredom of workers for the candidate workcell operation

4: Resistance of workers to learn programming languages

5: Resistance of maintenance workers to learn cobot system maintenance

6: Ability of maintenance workers to learn to work with the cobot

7: Availability of local maintenance/service experts

8: Availability of cobot system integrators

9: Distributers and resellers are often motivated to help and perform demos

10: Type and level of skill gaps in current workforce ${ }^{17}$

11: Workers union resistance

D: Key elements for financial

1: Lifecycle costs of cobot with human compared to a human worker alone

2: SMM's budgeted amount for the whole cobot integration project compared to the prorated cost of the total cobot system project.

3: SMM's level of risk tolerance

4: Time to full ROI (return on investment)

- When generating the ROI, make sure it is done holistically, which may imply that using cobots may not the best solution, or may reveal that a manual solution or non-robotic automation solution will be the faster, better, cheaper, and safer solution

- Common experience reported by seasoned cobot system integrators ${ }^{18}$

- ROI can typically be reached within 14 months

- SMMs should budget around 3 times the price of the cobot system to cover integration costs, however, some MEP experts have experienced as little as $1 / 2$ to 1 times the cost of cobot for development, fixturing, end effectors, and deployment

5: Availability and variety of leasing options and payment plans

6: Product bundles availability

7: Availability of application-specific packages for the cobot system

E: Miscellaneous key elements

1: Study what the SMM's business is, study each workcell and start with a simpler workcell operations since there is a significant non-technical danger in picking the "wrong" workcell

2: Use "right and ready" assessment document from South Dakota MEP

3: Favor starting with a simpler workcell operation since there is a significant non-technical danger in picking the "wrong" workcell

4: Part of a community of users/researchers

5: Process variability ${ }^{19}$ in each workcell ${ }^{20}$

6: Product characteristic variability ${ }^{21}$

7: Ensure that enough monies are available to be allocated before deploying the cobot

8: Cobot system has been "mainstreamed," or widely used

9: Availability of tooling and accessory options

10: Scalability requirements to support automation growth over the long term

\footnotetext{
${ }^{17}$ A higher level of skill gap in the plant's workforce implies greater benefit with cobot integration. Rough estimates are OK, just be consistent from use case to use case.

${ }^{18}$ South Dakota MEP

${ }_{19}$ Process "variability" means the number of process changes and the magnitude of those process changes that the workcell process must go through

${ }^{20}$ Workcells with low variability are generally preferred to workcells with highly variable processes

${ }^{21}$ Product characteristic variability means that the type of part dimensions, surface attributes (e.g., finish), and possibly pose changes non-trivially and frequently
} 
11: Cost of delaying moving to cobot technology 22

12: Compliant to standards

${ }^{22}$ Some MEP experts argue that the use of collaborative robots now is like that of adopting early computers, which were initially slow and fault ridden, but those who adopted computers early had the advantage of experience for the later benefits of computer technology. In conclusion, the SMM must consider the cost of delaying moving to cobot technology. 
Appendix B: An example case study for the Key-elements weighting approach

We now propose an example case study for conducting a key-elements weighting approach for choosing the best workcell for cobot integration.

Say that we have a SMM with only two workcells that would be a candidate for cobot integration, namely, Machine Tending and Assembly, and the SMM needs to know which workcell among these two would supply the greater benefit to the company. Appropriate experts make assessments by examining each of the elements for both the Machine Tending use case and the Assembly use case. Assuming the experts choose to use all the suggested elements, the experts would come up with assessments for each of the 51 elements in Appendix A for each use case. For example, each key element may be scored with a weighting of -1, 0, 1, or 2 , where a -1 weighting means that key element is detrimental, and a +2 weighting means the key element is of high importance.

Let's propose that in our example case study the group came up with the following sums of the 51 scores: 36 for Machine Tending 17 for Assembly

In this example case study, the group found that integrating a cobot into the Machine Tending workcell (or workcells) would likely provide the best ROI. 


\section{List of Figures}

Figure 1: Robot-integrated workcell types with percent benefit to the manufacturers ......................................... 3

Figure 2: Complexity-impact quadrant .......................................................................................................... 3

\section{References}

- Consider the following popular resources for cobots ${ }^{23}$ :

○ https://robotiq.com/resource-center

- https://robotiq.com/resource-center/ebooks/

○ https://robotiq.com/resource-center/case-studies

○ https://blog.robotiq.com/collaborative-robot-ebook

○ https://leanrobotics.org/resources/

○ https://www.robotics.org/Robotic-Resources

$\circ$ https://www.mmsonline.com/zones/Robots-and-Automation

${ }^{23}$ Certain commercial equipment or materials are identified in this paper in order to specify the procedure adequately. Such identification is not intended to imply recommendation or endorsement by NIST, nor is it intended to imply that the materials or equipment identified are necessarily the best available for the purpose. 